\title{
Mechanisms of xylem hydraulic recovery after drought in Eucalyptus saligna
}

\author{
Alice Gauthey ${ }^{1}$, Jennifer Peters ${ }^{2}$, Rosana López ${ }^{3}$, Madeline Carins Murphy $^{4}$, Celia M. \\ Rodriguez-Dominguez ${ }^{5}$, David Tissue ${ }^{1}$, Belinda Medlyn ${ }^{6}$, Timothy Brodribb ${ }^{4}$, and Brendan \\ Choat $^{1}$ \\ ${ }^{1}$ Western Sydney University \\ ${ }^{2}$ Western Sydney University Hawkesbury Institute for the Environment \\ ${ }^{3}$ Universidad Politécnica de Madrid \\ ${ }^{4}$ University of Tasmania \\ ${ }^{5}$ Instituto de Recursos Naturales y Agrobiología de Sevilla \\ ${ }^{6}$ Macquarie University
}

May 3, 2021

\begin{abstract}
The mechanisms by which woody plants recover xylem hydraulic capacity after drought stress are not well understood, particularly with regard to the role of embolism refilling. We evaluated the recovery of xylem hydraulic capacity in young Eucalyptus saligna plants exposed to cycles of drought stress and rewatering. Plants were exposed to moderate and severe drought stress treatments, with recovery monitored at time intervals from $24 \mathrm{hrs}$ to 6 months after rewatering. The percentage loss of xylem vessels due to embolism (PLV) was quantified at each time point using micro-computed tomography with stem water potential $\left(\Psi_{\mathrm{x}}\right)$ and whole plant transpiration (Eplant) measured prior to scans. Plants exposed to severe drought stress suffered high levels of embolism $(47.38 \pm 10.97 \%$ PLV $)$ and almost complete canopy loss. No evidence of embolism refilling was observed at $24 \mathrm{hrs}$, one week, or three weeks after rewatering despite rapid recovery in $\Psi \mathrm{x}$. Recovery of hydraulic capacity was achieved over a 6-month period by growth of new xylem tissue, with canopy leaf area and Eplant recovering over the same period. These findings indicate that E. saligna recovers slowly from severe drought stress, with potential for embolism to persist in the xylem for many months after rainfall.
\end{abstract}

\section{Hosted file}

AGauthey_Recovery_Main_document_final_PCE.pdf available at https://authorea.com/users/411570/ articles/520547-mechanisms-of-xylem-hydraulic-recovery-after-drought-in-eucalyptussaligna 